\title{
Prospects of Spin Catalysis on Spin-Polarized Graphene Heterostructures
}

\author{
Pavel V. Avramov, ${ }^{\mathrm{A}, \mathrm{E}}$ Pavel B. Sorokin, ${ }^{\mathrm{B}}$ Alex A. Kuzubov, ${ }^{\mathrm{C}}$ Seiji Sakai, ${ }^{\mathrm{D}}$ \\ Shiro Entani, ${ }^{\mathrm{D}}$ and Hiroshi Naramoto ${ }^{\mathrm{D}}$ \\ A Kyungpook National University, 1370 Sankyuk-dong, Buk-gu, Daegu, 702-701, \\ Republic of Korea. \\ ${ }^{B}$ National University of Science and Technology MISiS, 4 Leninskiy prospekt, Moscow, \\ 119049, Russian Federation. \\ C Siberian Federal University, 79 Svobodniy av., Krasnoyarsk 660041, \\ Russian Federation. \\ ${ }^{D}$ Advanced Science Research Center, Japan Atomic Energy Agency, 2-4 Shirakata \\ Shirane, Tokai-mura, Naka-gun, Ibaraki-ken 319-1195, Japan. \\ ${ }^{\mathrm{E}}$ Corresponding author. Email: paul@iph.krasn.ru
}

\begin{abstract}
Extreme points on potential energy surfaces of Ni adatom on free-standing graphene and top:fcc and $h c p: f c c$ graphene/ $\mathrm{Ni}(111)$ heterostructures in different spin states were studied using periodic boundary conditions density functional theory approach. It was found that the spin states of the substrates strongly influence the energy of the Ni adatom extreme points on potential energy surface by decreasing (top: $f c c$ heterostructure) or increasing ( $h c p: f c c$ heterostructure) the total energies of $\eta^{1}, \eta^{1^{\prime}}$, and $\eta^{2} \mathrm{Ni}$ adatom coordinations on graphene. This phenomenon offers unique possibilities to control the potential energy surfaces of transition metal adatoms and promote surface chemical reactions using induced spin polarization of graphene substrates.
\end{abstract}

Manuscript received: 10 April 2015.

Manuscript accepted: 12 November 2015.

Published online: 15 December 2015.

\section{Introduction}

The activity and selectivity of transition metal nanocluster (nc-TM) catalysis can be controlled by tailoring the size and shape of the metal nanoparticles ${ }^{[1-3]}$ by deposition of TM atoms onto templates suitable for nucleation. Graphene and h-BN nanomeshes on metal surfaces have recently been considered as successful candidates for nc-TM nucleation due to their periodic corrugated topography. ${ }^{[4-20]}$ The potential energy surfaces of a set of TM nanoclusters on graphene/ $\mathrm{Rh}(111)$, graphene/ $\mathrm{Ru}(0001)$, and h-BN/Ru(0001) were studied by density functional theory (DFT) calculations. ${ }^{[21]}$ It was demonstrated that these supports provide different template functionalities ranging from zero-dimensional TM clusters to two-dimensional (2D) networks for adsorbates.

It was shown ${ }^{[22]}$ that the formation of hexahapto-coordinated metal ions provides new possibilities to develop new-era lowdimensional graphene and carbon nanotube materials for organometallic catalysis and spintronics by preserving the graphitic band structure during formation of bis-hexahapto-metal bonds. It was shown that a network ${ }^{[23]}$ of single $\mathrm{Cr}$ atoms with arene ligands trapped in a periodic potential well of a graphene layer opens new possibilities to quantum routeing and state manipulation through the conductive graphene substrate. It was found that ferromagnetic ordering in the ground state is characterized by a high magnetization energy of $400 \mathrm{meV}$. Spin-carrying hexahapto-coordinated isolated $\mathrm{Cr}$-arene groups interact with each other via a spin-communication net within the graphene layer. The effect was interpreted in terms of spin-charge separation induced by $\pi-\mathrm{d}$ interactions.

The catalytic activity of several $\mathrm{TM}_{n}(\mathrm{TM}=\mathrm{Ti}, \mathrm{Au}, \mathrm{Pt}, \mathrm{Ag}$, $\mathrm{Al}, \mathrm{Pd}, \mathrm{Cu} ; n=1,7,8,9,13,16)$ clusters embedded into or deposited on pristine, ${ }^{[24]}$ defect, ${ }^{[25]}$ or strained ${ }^{[26]}$ graphene was studied using $a b$ initio DFT calculations. It was shown that embedded TM atoms significantly enhance chemical interactions between graphene and $\mathrm{O}_{2}, \mathrm{CO}, \mathrm{NO}_{2}$, and $\mathrm{NH}_{3}$ molecules. $\mathrm{TM}_{n}$-derived catalytic oxidation of $\mathrm{CO}$ can be greatly enhanced by the presence of graphene defects or structural strain. An Aumodified graphene sensor for $\mathrm{H}_{2} \mathrm{~S}$ and $\mathrm{SOF}_{2}$ molecules was developed using a combined experimental and theoretical DFT approach. ${ }^{[27]}$ The ab initio DFT approach was employed to simulate the interactions of $\mathrm{H}_{2} \mathrm{~S}$ and $\mathrm{SOF}_{2}$ molecules with $\mathrm{Au}$ nanoclusters deposited on graphene and to interpret experimental data. The density of states and Mulliken population analysis confirmed experimentally observed charge transfer in nc-Auderived chemisorption.

As the deposition of graphene on ferromagnetic substrates such as $\mathrm{Ni}(111)$ causes subsequent visible changes in the electronic structure of the nanocarbon fragment due to spin polarization (see, for example ref. 28), we can expect some chemical reactions to proceed via spin-dependent mechanisms e.g. TM clusterization on graphene, which is actually a subject of spin-catalytic effect (see, for example ref. 29). This effect is caused by the spin conversion of the reagents in the presence of the spin catalyst with uncompensated spin; two main examples 
of such phenomenon are the photolysis of diphenylpentanone and the recombination of triplet pair of two benzyl radicals during photolysis of dibenzylketone after decarbolination of the initial pair.

The main goal of this paper is to study the qualitative dependence of extreme points of potential energy surfaces of $\mathrm{Ni}$ adatom on graphene/ $\mathrm{Ni}(111)$ heterostructures upon the spin states of $\mathrm{Ni}(111)$ substrate. It was found that the extreme points on the potential energy surfaces are strongly influenced by the spin polarization of graphene caused by the spin state of the nickel support. This finding opens a challenging opportunity to employing graphene layers deposited on ferromagnetic supports as templates for spin-catalyzed reactions.

\section{Computational Methods}

The Ni(111) substrate is one of the best choices to deposit graphene ${ }^{[30]}$ due to close match of the crystalline lattices. Among several possible graphene/Ni(111) interfaces, the top: $f c c$ (carbon atoms are placed atop of top and $f c c$ positions of nickel lattice) and $h c p$ : $f c c$ (carbon atoms are placed atop of $h c p$ and $f c c$ positions) configurations have been reported experimentally ${ }^{[31,32]}$ to result in the lowest and highest energy chemi- and physisorbed graphene/ $\mathrm{Ni}(111)$ heterostructures, respectively. The electronic structure calculations ${ }^{[33-37]}$ confirmed the experimental findings. The deposition of graphene on ferromagnetic supports (in particular on $\mathrm{Ni}(111)$ surface) leads to $\mathrm{C} 2 \mathrm{p}_{z}$-states spin polarization (see, for example, ${ }^{[28,38]}$ ) and some hybridization between graphene $\pi$ - and $\mathrm{Ni} 3 \mathrm{~d}$ valence bands.

For electronic structure calculations the $(4 \times 4)$ supercell of graphene/ $\mathrm{Ni}(111)$, consisting of 32 carbon atoms and a threelayered nickel plate (48 $\mathrm{Ni}$ atoms) were used for periodic boundary conditions (PBC) calculations of ferromagnetic and diamagnetic spin states, taking into account the spin-orbital interactions. Both the chemisorbed graphene/Ni(111) top: $f c c$ (displaying the lowest energy) and physisorbed hcp:fcc (displaying the highest energy) configurations were used to study the potential energy surfaces of nickel adatom migration on graphene. The Ni adatom was placed on $\eta^{1}$ (atop carbon atoms in $f c c$ positions), $\eta^{1^{\prime}}$ (atop carbon atoms in top or $h c p$ positions for top: $f c c$ and $h c p: f c c$ heterostructures, respectively), $\eta^{2}$ (atop the $\mathrm{C}-\mathrm{C}$ bond), and $\eta^{6}$ (atop of the centre of a carbon hexagon at $h c p$ and $f_{c p}$ positions of the nickel lattice) positions. As a reference, a free-standing graphene with single nickel adatom atop the same positions was examined in both ferromagnetic and diamagnetic spin states by artificially switching the spin state in the course of the calculations (Fig. 1).

For weakly bound periodic multilayered 2D heterostructures, an accurate consideration of weak dispersion forces (see for example ${ }^{[39]}$ ) plays an essential role in the determination of the interface structures. Two distinctively different DFT approaches are used to calculate the atomic and electronic structures of graphene-based heterostructures. The first approach is based on long-corrected generalized-gradient approach (LC GGA) DFT approximation, with empirical consideration of dispersion forces (see for example $e^{[40-42]}$ ). This approach reproduces the atomic structure of weakly bound graphene-based 2D heterostructures with reasonable accuracy. The second approach is based on a less sophisticated, however, very practical local-density approximation (LDA). The LDA is the simplest form of DFT and suffers from lack of accuracy in the determination of bandgap width of semiconducting materials; however, the LDA works surprisingly well in the determination of the atomic structure of weakly bonded carbon nanostructures (e.g. fullerites, heterostructures of carbon nanotubes, graphite $)^{[43-48]}$ due to artificial cancelation of fundamental disadvantages of the LDA theory.

The electronic structure calculations were performed using $\mathrm{DFT}^{[49,50]}$ within the LDA for the exchange-correlation functional ${ }^{[51]}$ with $\mathrm{PBC}$ using Vienna ab initio simulation package. ${ }^{[52-54]}$ The projector augment wave method along with a plane wave basis set with an energy cut-off of $400 \mathrm{eV}$ were used. To calculate equilibrium atomic structures, the Brillouin zone was sampled according to the Monkhorst-Pack ${ }^{[55]}$ scheme with $8 \times 8 \times 1 k$-points mesh. The density of states was calculated using $14 \times 14 \times 1 \mathrm{k}$-points mesh and energy smearing factor of $0.2 \mathrm{eV}$. To avoid spurious interactions between neighbouring structures in a hexagonal supercell, a vacuum layer of $10 \AA$ in non-periodic direction was included. The extreme points on the potential energy surfaces were located by structural optimization with different starting geometries. Structural relaxation was performed until the forces acting on each atom were less $0.05 \mathrm{eV} \AA^{-1}$. The hexagonal unit cell of graphene was (a) Ni adatom on free-standing graphene, $\eta^{1}, \eta^{2}$, and $\eta^{6}$ configurations

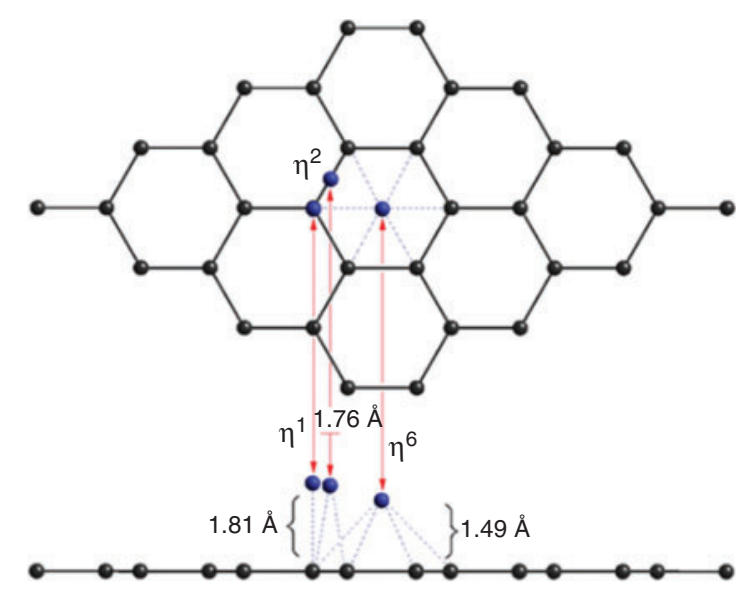

(b) $\mathrm{Ni}$ adatom on top : fcc graphene/Ni(111) $\eta^{1} \eta^{1^{\prime}}$ configurations

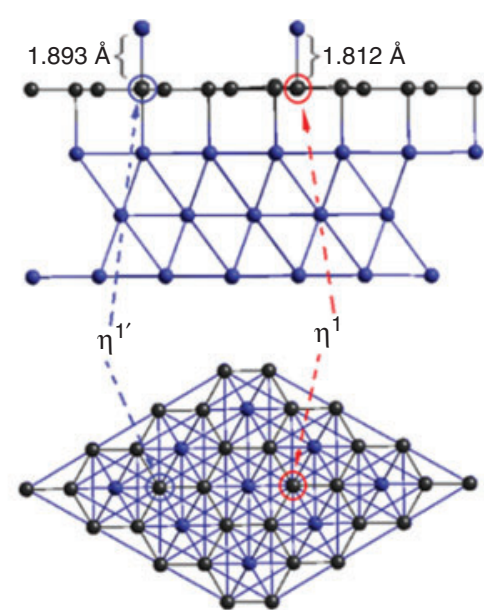

Fig. 1. (a) Atomic structure of $\mathrm{Ni}$ adatom on free-standing graphene in $\eta^{1}, \eta^{2}$, and $\eta^{6}$ configurations. (b) Ni adatom on graphene (top:fcc)/Ni(111) heterostructure in $\eta^{1}$ and $\eta^{1^{\prime}}$ configurations. 
multiplied $4 \times 4$ times in order to avoid interaction between neighbouring $\mathrm{Ni}$ adatoms. The spin-orbital coupling was taken into account according to ref. 56.

A brief survey of the publications in the field of electronic structure calculations of weakly bound graphene-metal heterostructures show that the LDA approach is frequently used to study graphene on metallic substrates ${ }^{[57-61]}$ because it predicts graphene-metal interfacial geometries in closer agreement with experimental data than does GGA approach. ${ }^{[62]}$ Even for a three-layered Ni plate, the LDA approach reproduces reasonably well the Ni-Ni distances in bulk nickel crystal $(2.422$ $2.423 \AA$ in comparison with the experimental value ${ }^{[63]}$ of $2.492 \AA)$. This result agrees $(2.427-2.442 \AA)$ with that obtained from the LDA calculations of graphene/ $\mathrm{Ni}(111)$ heterostructures for nine-layered Ni(111) plate. ${ }^{[28]}$ The graphene-Ni(111) distances for the top: $f c c$ and $h c p: f c c$ configurations are 2.08 and $3.21 \AA$, respectively, in comparison with the experimental ${ }^{[64,65]}$ (2.14 and $2.80 \AA$, respectively), Perdew Burke Ernzerhof DFT $(\mathrm{PBE})^{[65,66]}(2.13$ and $3.05 \AA$, respectively), and PBE taking into account van der Waals terms $(\mathrm{vdW})^{[67]}(2.12$ and $3.76 \AA$, respectively) data. Previous LDA calculations ${ }^{[28,68]}$ of both configurations give very similar graphene- $\mathrm{Ni}(111)$ distances $(2.06$, 3.62 and $2.04,3.24 \AA$, respectively). Our calculation model qualitatively reproduces the experimental atomic structure and agrees with previous electronic structure calculations.

The binding energies $E_{\mathrm{B}}$ of $\mathrm{Ni}$ adatoms on the substrates were calculated for both diamagnetic and ferromagnetic states using the following expression:

$$
E_{\mathrm{B}}=E_{\mathrm{h}-\mathrm{Ni}}-E_{\mathrm{h}}-E_{\mathrm{Ni}}
$$

where $E_{\mathrm{h}-\mathrm{Ni}}$ is total energy of the heterostructure with adsorbed $\mathrm{Ni}$ adatom, $E_{\mathrm{h}}$ is total energy of the heterostructure without $\mathrm{Ni}$ adatom, and $E_{\mathrm{Ni}}$ is total energy of $\mathrm{Ni}$ atom.

\section{Results and Discussion}

The atomic structure, and binding and relative energies of global $\eta^{6}$ and local $\eta^{1}$ and $\eta^{2}$ minima of Ni adatom on pristine graphene for both diamagnetic and ferromagnetic spin states are presented in Table 1. For both spin states, the $\eta^{6}$ configurations

Table 1. Details of atomic structure, and relative $(\Delta E)$, and binding $\left(E_{\mathrm{B}}\right)$ energies of $\mathrm{Ni}$ adatom on pristine graphene in spin-unpolarized diamagnetic and spin-polarized ferromagnetic states

\begin{tabular}{|c|c|c|c|c|c|c|}
\hline \multirow[t]{2}{*}{$\mathrm{Ni}$ adatom } & \multicolumn{3}{|c|}{ Diamagnetic state } & \multicolumn{3}{|c|}{ Ferromagnetic state } \\
\hline & $\begin{array}{c}\Delta E \\
{[\mathrm{eV}]}\end{array}$ & $\begin{array}{c}d_{\mathrm{Ni}-\text { graphene }} \\
{[\AA]}\end{array}$ & $\begin{array}{c}E_{\mathrm{B}} \\
{[\mathrm{eV}]}\end{array}$ & $\begin{array}{c}\Delta E \\
{[\mathrm{eV}]}\end{array}$ & $\begin{array}{c}d_{\mathrm{Ni} \text {-graphene }} \\
{[\AA]}\end{array}$ & $\begin{array}{c}E_{\mathrm{B}} \\
{[\mathrm{eV}]}\end{array}$ \\
\hline$\eta^{6}$ & 0.0 & 1.49 & -3.44 & 0.0 & 1.49 & -3.44 \\
\hline$\eta^{1}$ & 0.9 & 1.81 & -2.54 & 0.9 & 1.81 & -2.54 \\
\hline$\eta^{2}$ & 0.6 & 1.76 & -2.86 & 0.7 & 1.76 & -2.76 \\
\hline
\end{tabular}

are the global minima with almost equal total energies (relative energies $E_{\mathrm{Ni} / \text { graphene }}^{\mathrm{DM}}=E_{\mathrm{Ni} / \text { graphene }}^{\mathrm{FM}}=0 \mathrm{eV}$; where $\mathrm{DM}=$ spinunpolarized diamagnetic and $\mathrm{FM}=$ spin-polarized ferromagnetic). The energy differences between $\eta^{6}$ and $\eta^{2}$ and $\eta^{1}$ extreme points for the FM states are 0.7 and $0.9 \mathrm{eV}$ per unit cell, respectively. And the energy differences between $\eta^{6}$ and $\eta^{2}$ and $\eta^{1}$ extreme points for the DM states are 0.6 and $0.9 \mathrm{eV}$ per unit cell, respectively. For the $\eta^{6}$ global minimum, the Ni adatomgraphene distance is $1.49 \AA$ for both spin states, whereas for $\eta^{2}$, the distance between adatom and graphene is $1.76 \AA$. The $\eta^{1}$ position is highest in energy, featuring a distance and a relative energy of $1.81 \AA$ and $0.9 \mathrm{eV}$, respectively, for both states. The binding energies of $\mathrm{Ni}$ adatom in different configurations are very close for $\eta^{6}$ and $\eta^{1}$ configurations and slightly different $(0.1 \mathrm{eV})$ for $\eta^{2}$.

The atomic structure and relative energies of $\eta^{6}$ minima and $\eta^{1}, \eta^{1^{\prime}}$, and $\eta^{2}$ extreme points on the potential energy surfaces of $\mathrm{Ni}$ adatom on graphene deposited on $\mathrm{Ni}(111)$ in $h c p: f c c$ position (graphene $(h c p: f c c) / \mathrm{Ni}(111)$ heterostructure) are presented in Table 2 and Fig. 1 for both FM and DM states. The FM graphene $(h c p: f c c) / \mathrm{Ni}(111)$ is metastable, with a relative energy of $2.15 \mathrm{eV}$ per supercell with respect to FM graphene(top: $f c c$ )/ $\mathrm{Ni}(111)$. The $\mathrm{Ni}$ adatom $\mathrm{FM} \eta^{6}$ is global minimum $(0.0 \mathrm{eV}$ relative energy), with a $2.58 \mathrm{eV}$ relative energy of $\mathrm{DM} \eta^{6}$. (It is worth noting that the relative energy of DM graphene( $h c p: f c c) /$ $\mathrm{Ni}(111)$ with respect to $\mathrm{FM}$ graphene $($ top: $f c c) / \mathrm{Ni}(111)$ is $4.73 \mathrm{eV}$.) Both $\eta^{6}$ coordinations display the same graphene$\mathrm{Ni}$ adatom bond length of $1.52 \AA$, with slightly longer $\mathrm{C}-\mathrm{C}$ bonds at the adsorption sites. DM $\eta^{1}, \eta^{1^{\prime}}$, and $\eta^{2} \mathrm{Ni}$ adatom coordinations are extreme points on the potential energy surface with comparable relative energies (i.e. 0.6, 0.6, and $0.5 \mathrm{eV}$, respectively) and graphene-Ni adatom distances (1.83, 1.83, and $1.77 \AA$, respectively). Spin polarization of the heterostructure leads to dramatic change in the structure of the complexes: the relative energy of $\eta^{1}$ coordination of $\mathrm{Ni}$ adatom increases from 0.6 (DM state) to $3.9 \mathrm{eV}$ (FM state), with very different $\mathrm{Ni}$ adatom-graphene distances (1.83 and $3.40 \AA$, respectively). Spin polarization of $\eta^{1^{\prime}}$ (relative energy of $0.6 \mathrm{eV}$ and graphene-Ni adatom distance of $1.83 \AA$ ) leads to transformation of the initial geometry to $\eta^{6}$ isomer. Finally, the $\eta^{2}$ coordination becomes an unbound state with a very high repulsive relative energy. The spin state of graphene $(h c p: f c c) / \mathrm{Ni}(111)$ substrate also influences the binding energies of $\mathrm{Ni}$ adatom on the heterostructure. Switching the spin polarization results in positive binding energies of the $\eta^{1}$ and $\eta^{2}$ coordinations, thus effectively ruling out the involvement of these coordination types in the migration phase of surface chemical reactions on graphene $(h c p: f c c) / \mathrm{Ni}(111)$ substrate.

The atomic structure, and binding and relative energies of $\eta^{6}$ minima, $\eta^{1}, \eta^{1^{\prime}}$, and $\eta^{2}$ extreme points on the potential energy surfaces of $\mathrm{Ni} /$ graphene $(t o p-f c c) / \mathrm{Ni}(111)$ heterostructure in both DM and FM states are presented in Table 3 and Fig. 1.

Table 2. Details of atomic structure, and relative $(\Delta E)$ and binding $\left(E_{\mathrm{B}}\right)$ energies of Ni adatom on graphene/Ni(111) (hcp:fcc configuration) in spin-unpolarized diamagnetic and spin-polarized ferromagnetic states

\begin{tabular}{|c|c|c|c|c|c|c|c|c|}
\hline \multirow[t]{2}{*}{ Ni adatom } & \multicolumn{4}{|c|}{ Diamagnetic state } & \multicolumn{4}{|c|}{ Ferromagnetic state } \\
\hline & $\Delta E[\mathrm{eV}]$ & $d_{\mathrm{Ni} \text {-graphene }}[\AA]$ & $d_{\text {graphene-Ni(111) }}[\AA]$ & $E_{\mathrm{B}}[\mathrm{eV}]$ & $\Delta E[\mathrm{eV}]$ & $d_{\text {Ni-graphene }}[\AA]$ & $d_{\text {graphene-Ni(111) }}[\AA]$ & $E_{\mathrm{B}}[\mathrm{eV}]$ \\
\hline$\eta^{6}$ & 0.0 & 1.52 & 3.20 & -3.11 & 0.0 & 1.52 & 3.20 & -2.92 \\
\hline$\eta^{1}$ & 0.6 & 1.83 & 3.20 & -2.55 & 3.9 & 3.40 & 3.22 & 0.96 \\
\hline$\eta^{1^{\prime}}$ & 0.6 & 1.83 & 3.20 & -2.55 & - & - & - & -2.38 \\
\hline$\eta^{2}$ & 0.5 & 1.77 & 3.22 & -2.59 & $>10$ & 3.40 & 3.22 & 83.07 \\
\hline
\end{tabular}


Table 3. Details of atomic structure, and relative $(\Delta E)$ and binding $\left(E_{\mathrm{B}}\right)$ energies of Ni adatom on graphene(top:fcc)/Ni(111) in diamagnetic and ferromagnetic spin states. The $\eta^{1^{\prime}}$ coordination corresponds to Ni adatom coordinated to a carbon atom atop of $f c c$ position of Ni plate

\begin{tabular}{|c|c|c|c|c|c|c|c|c|}
\hline \multirow[t]{2}{*}{$\mathrm{Ni}$ adatom } & \multicolumn{4}{|c|}{ Diamagnetic state } & \multicolumn{4}{|c|}{ Ferromagnetic state } \\
\hline & $\Delta E[\mathrm{eV}]$ & $d_{\mathrm{Ni}-\text { graphene }}[\AA]$ & $d_{\text {graphene-surface }}[\AA]$ & $E_{\mathrm{B}}[\mathrm{eV}]$ & $\Delta E[\mathrm{eV}]$ & $d_{\mathrm{Ni}-\text { graphene }}[\AA]$ & $d_{\text {graphene-surface }}[\AA]$ & $E_{\mathrm{B}}[\mathrm{eV}]$ \\
\hline$\overline{\eta^{6}}$ & 0.0 & 1.56 & 2.04 & -2.93 & 0.0 & 1.55 & 2.07 & -2.69 \\
\hline$\eta^{1}$ & 2.9 & 2.98 & 2.00 & $>0$ & 0.4 & 1.81 & 2.01 & -2.28 \\
\hline$\eta^{1^{\prime}}$ & 0.1 & 1.56 & 2.04 & -2.80 & 0.2 & 1.56 & 2.08 & -2.52 \\
\hline$\eta^{2}$ & 0.1 & 1.76 & 2.03 & -2.81 & 0.1 & 1.77 & 2.07 & -2.56 \\
\hline
\end{tabular}

$\mathrm{Ni}$ adatom and graphene PDOS of Ni/graphene

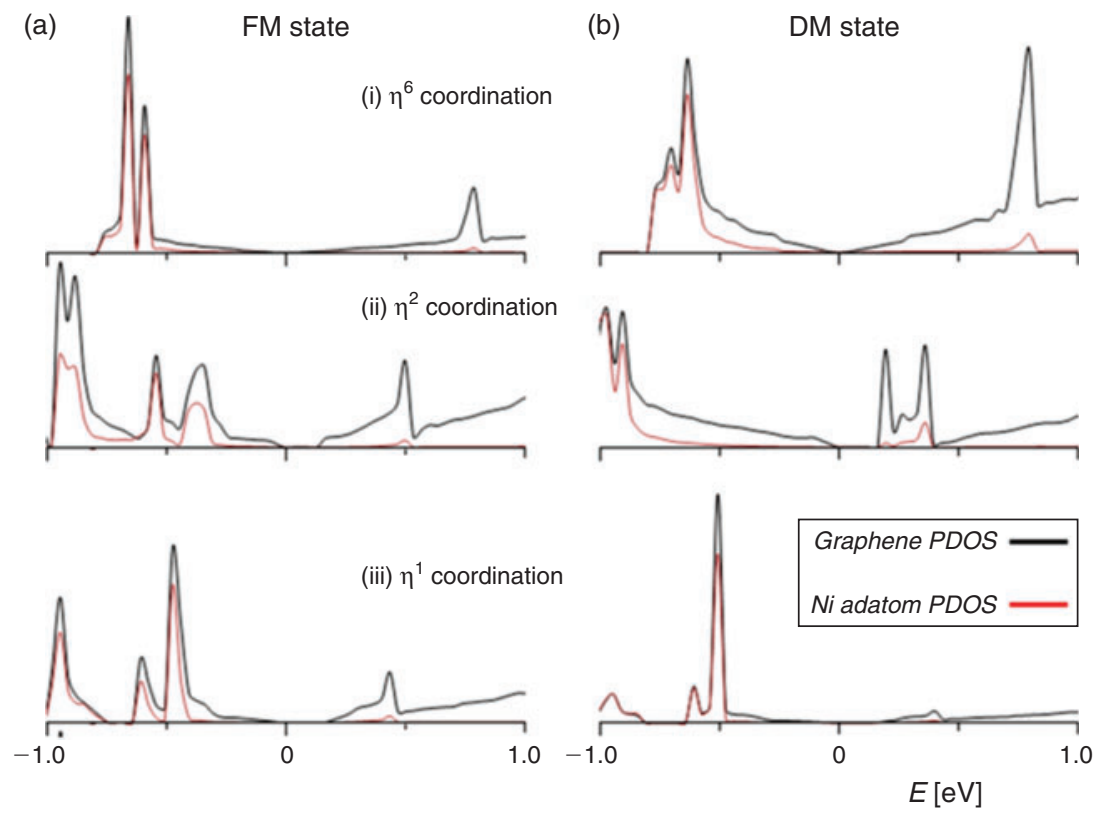

Fig. 2. Partial density of states of Ni adatom/graphene heterostructure in (a) FM and (b) DM states for $\eta^{6}$ (i), $\eta^{2}$ (ii), and $\eta^{1}$ (iii) configurations. The graphene and Ni PDOS data are shows as the black and red curves, respectively. The Fermi energy is taken as zero.

The energy splitting between the global $\eta^{6}$ coordinations of the $\mathrm{FM}$ and DM states is $1.62 \mathrm{eV}$, making FM $\eta^{6}$ coordination the global minimum of the heterostructure. The DM $\eta^{1}$ coordination is the highest in energy (relative energy of $2.9 \mathrm{eV}$ and $\mathrm{Ni}$ adatom-graphene distance of $2.98 \AA$ ), and $\eta^{1^{\prime}}$ and $\eta^{2}$ extreme points both feature a relative energy of $0.1 \mathrm{eV}$ and comparable adatom-graphene distances (1.56 and $1.76 \AA$, respectively). Switching the spin polarization of graphene dramatically changes the characteristics of the extreme points of $\mathrm{Ni}$ adatom potential energy surface on graphene $($ top $-f c c) / \mathrm{Ni}(111)$ : the $\eta^{1}$ local minimum displayed significant reduction in both the energy (from 2.9 to $0.4 \mathrm{eV}$ ) and $\mathrm{Ni}$ adatom-graphene distance (from 2.98 to $1.81 \AA$ ). These values are comparable with those displayed by the $\eta^{1^{\prime}}$ and $\eta^{2}$ states. The binding energies of $\mathrm{Ni}$ adatom strongly depend on the spin state of the support: spin polarization makes $\eta^{1}$ and $\eta^{2}$ coordinations energetically unfavourable sites and slightly $(\sim 0.2 \mathrm{eV})$ increases the binding energies for $\eta^{6}$ and $\eta^{1^{\prime}}$ coordinations.

The partial density of states (PDOS) of $\mathrm{Ni}$ adatom and graphene of $\mathrm{Ni}$ /graphene heterostructures in DM and FM states are presented in Fig. 2. The graphene PDOS of global minimum $\eta^{6}$ coordination displays a typical Dirac cone in the vicinity of the Fermi level with a sharp peak at $-0.798 \mathrm{eV}$ for both FM and DM states. The local $\eta^{1}$ and $\eta^{2}$ minima clearly display complete destruction of the Dirac cone structure for both spin states due to re-hybridization of the carbon atoms chemically connected to Ni.

The total density of states (TDOS) and PDOS of Ni/graphene $(h c p: f c c) / \mathrm{Ni}(111)$ with different $\mathrm{Ni}$ adatom coordinations $\left(\eta^{6}\right.$, $\eta^{2}, \eta^{1}$, and $\eta^{1^{\prime}}$ ) and spin states are presented in Fig. 3. Both FM and $\mathrm{DM}$ states of all the $\mathrm{Ni}$ adatom coordinations display complete destruction of the Dirac cone in the vicinity of the Fermi level, with strong spin polarization of graphene and $\mathrm{Ni}$ adatom PDOS. In the vicinity of the Fermi level, spin polarization completely alters the graphene electronic structure, thereby considerably changing the structure and properties of critical points on the potential energy surface of graphene by increasing the relative energy of $\eta^{1}$ coordination from 0.6 to $3.9 \mathrm{eV}$ and making $\eta^{2}$ coordination an unbound state (Table 2). This result suggests that migration of $\mathrm{Ni}$ adatoms is spin-dependent, and therefore it is possible to control the formation of metal nanostructures on graphene by using appropriate TM substrates. It is particularly intriguing in view of the importance of isolating metal adatoms on graphene that aggregation leads to the significant degradation of magnetic, emission, or even sorption $^{[69]}$ properties.

The TDOS and PDOS of Ni/graphene(top: $f c c) / \mathrm{Ni}(111)$ heterostructure with different $\mathrm{Ni}$ adatom coordinations $\left(\eta^{6}, \eta^{2}, \eta^{1}\right.$, and $\left.\eta^{1^{\prime}}\right)$ and spin states are presented in Fig. 4. As in the hcp:fcc 


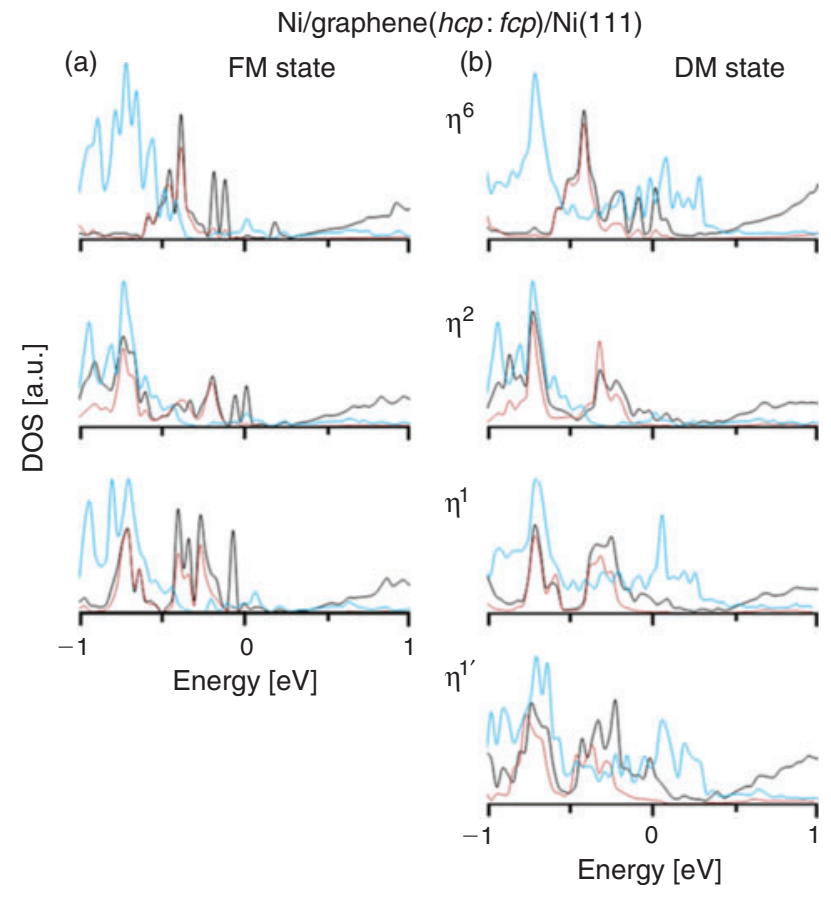

Fig. 3. Partial density of states of Ni/graphene $(h c p: f c c) / \mathrm{Ni}(111)$ heterostructure in (a) FM and (b) DM states for $\eta^{6}, \eta^{2}, \eta^{1}$, and $\eta^{1^{\prime}}$ coordinations of $\mathrm{Ni}$ adatom. The graphene, Ni adatom, and Ni plate PDOS data are shown as the black, red, and blue curves, respectively. The Fermi energy is taken as zero.

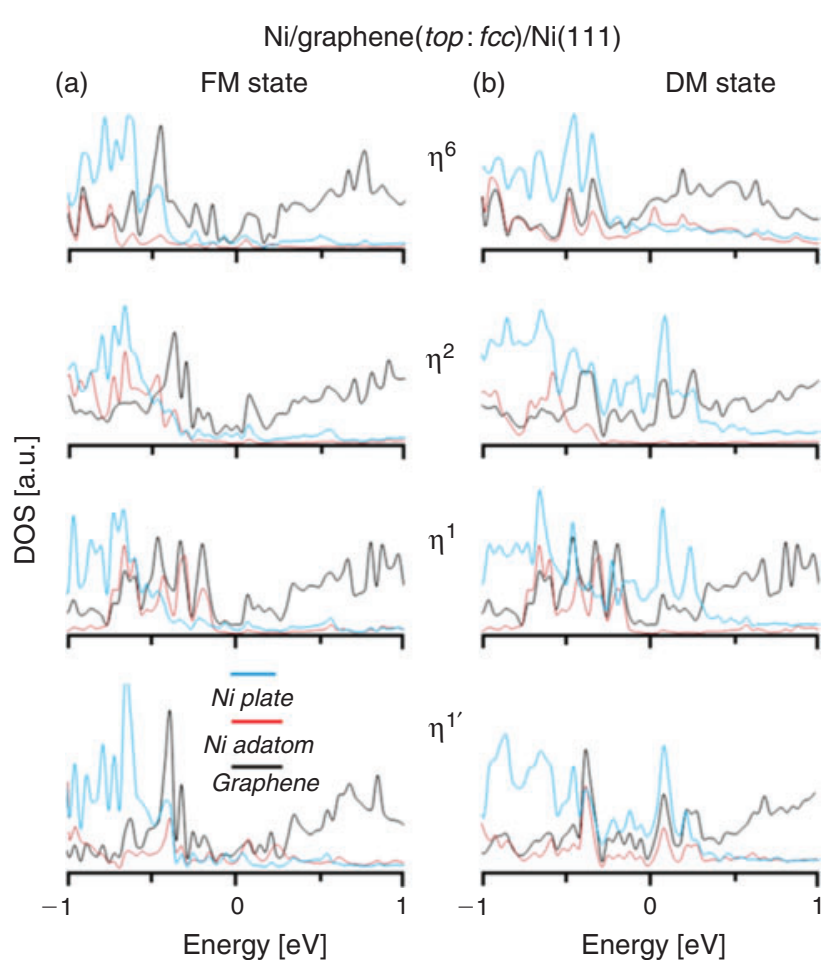

Fig. 4. Partial density of states of Ni/graphene(top: $f c c) / \mathrm{Ni}(111)$ heterostructure in (a) FM and (b) DM states for $\eta^{6}, \eta^{2}, \eta^{1}$, and $\eta^{1^{\prime}}$ coordinations of $\mathrm{Ni}$ adatom. The graphene, Ni adatom, and Ni plate PDOSes data are shows as the black, red, and blue curves, respectively. The Fermi energy is taken as zero. case, both FM and DM configurations of all the Ni adatom coordinations display complete destruction of the Dirac cone electronic structure. Inclusion of the spin polarization completely changes the PDOS of all $\eta^{6}, \eta^{2}, \eta^{1}$, and $\eta^{1^{\prime}}$ coordinations, causing fundamental changes in the potential energy surface of $\mathrm{Ni}$ adatom on graphene $($ top $: f c c) / \mathrm{Ni}(111)$ heterostructure (Table 3).

\section{Conclusions}

Electronic structure calculations of the $\mathrm{Ni} /$ graphene/Ni(111) $h c p: f c c$ and top: $f c c$ heterostructures qualitatively reveal significant changes in the potential energy surfaces of $\mathrm{Ni}$ adatom migration pathways on graphene based on the spin state of the heterostructures. As graphene either does not chemically interact with $\mathrm{Ni}(111)$ (hcp:fcc coordination) or reveal weak chemical interactions with the substrate (top: $f c c$ configuration), the significant changes in the graphene-Ni adatom potential energy surface upon changes in the spin states are determined mainly by the exchange interactions between the nickel substrate and graphene, which are the subject of spin catalytic effects. The direct Ni substrate-graphene exchange dramatically alters the graphene density of states and consequently affects the parameters of critical points on the potential energy surfaces of graphene. As a result, it may determine the migration pathways of $\mathrm{Ni}$ adatoms on graphene. The effect of spin heterogenic catalysis on spin-polarized graphene can be used to control the surface spin-selective chemical reactions, such as clusterization of transition metal clusters or formation of nanocluster superlattices on graphene, by switching the degree of spin polarization using different types of magnetic and non-magnetic supports.

\section{Acknowledgements}

We are grateful to the Joint Supercomputer Center of the Russian Academy of Sciences, and 'Lomonosov' supercomputers of Moscow State University for providing the chance of using a cluster computer for quantum-chemical calculations. Part of the calculations were made on the supercomputer cluster 'Cherry' provided by the Materials Modeling and Development Laboratory at NUST 'MISIS' (supported by a Grant from the Ministry of Education and Science of the Russian Federation No. 14.Y26.31.0005). P.V. A. and P.B.S. acknowledge Japan Atomic Energy Agency Advanced Science Research Center and Molecular Spintronics Group for hospitality and fruitful collaboration. The SFU team acknowledges support from the Russian Science Foundation (Project No. 14-13-00139). P.B.S. gratefully acknowledges the financial support of the Ministry of Education and Science of the Russian Federation in the framework of Increase Competitiveness Program of NUST 'MISIS' (No. K2-2015-033).

\section{References}

[1] I. Lee, F. Delbecq, R. Morales, M. A. Albiter, F. Zaera, Nat. Mater. 2009, 8, 132. doi:10.1038/NMAT2371

[2] F. J. Zaera, Phys. Chem. Lett. 2010, 1, 621. doi:10.1021/JZ9002586

[3] M. Valden, X. Lai, D. W. Goodman, Science 1998, 281, 1647. doi:10.1126/SCIENCE.281.5383.1647

[4] A. T. N'Diaye, S. Bleikamp, P. J. Feibelman, T. Michely, Phys. Rev. Lett. 2006, 97, 215501. doi:10.1103/PHYSREVLETT.97.215501

[5] D. G. Castner, B. A. Sexton, G. A. Somorjai, Surf. Sci. 1978, 71, 519. doi:10.1016/0039-6028(78)90444-2

[6] A. B. Preobrajenski, M. L. Ng, A. S. Vinogradov, N. Mårtensson, Phys. Rev. B 2008, 78, 073401. doi:10.1103/PHYSREVB.78.073401

[7] F. Müller, H. Sachdev, S. Hüfner, A. J. Pollard, E. W. Perkins, J. C. Russell, P. H. Beton, S. Gsell, M. Fischer, M. Schreck, Small 2009, 5 , 2291. doi:10.1002/SMLL.200900158

[8] B. Wang, M. Caffio, C. Bromley, H. Früchtl, R. Schaub, ACS Nano 2010, 4, 5773. doi:10.1021/NN101520K 
[9] M.-C. Wu, Q. Xu, D. W. Goodman, J. Phys. Chem. 1994, 98, 5104. doi:10.1021/J100070A027

[10] S. Marchini, S. Günther, J. Wintterlin, Phys. Rev. B 2007, 76, 075429. doi:10.1103/PHYSREVB.76.075429

[11] A. L. Vázquez de Parga, F. Calleja, B. Borca, M. C. G. Passeggi Jr, J. J. Hinarejos, F. Guinea, R. Miranda, Phys. Rev. Lett. 2008, 100, 056807. doi:10.1103/PHYSREVLETT.100.056807

[12] Y. Pan, H. Zhang, D. Shi, J. Sun, S. Du, F. Liu, H.-J. Gao, Adv. Mater. 2009, 21, 2777. doi:10.1002/ADMA.200800761

[13] P. W. Sutter, J.-I. Flege, E. A. Sutter, Nat. Mater. 2008, 7, 406. doi:10.1038/NMAT2166

[14] J. Wintterlin, M.-L. Bocquet, Surf. Sci. 2009, 603, 1841. doi:10.1016/ J.SUSC.2008.08.037

[15] M. L. Bocquet, B. Wang, Prog. Surf. Sci. 2010, 85, 435. doi:10.1016/ J.PROGSURF.2010.09.001

[16] M. Corso, W. Auwärter, M. Muntwiler, A. Tamai, T. Greber, J. Osterwalder, Science 2004, 303, 217. doi:10.1126/SCIENCE. 1091979

[17] A. Goriachko, Y. He, M. Knapp, H. Over, M. Corso, T. Brugger, S. Berner, J. Osterwalder, T. Greber, Langmuir 2007, 23, 2928. doi:10.1021/LA062990T

[18] A. Goriachko, Y. B. He, H. J. Over, PhysChem Comm 2008, $112,8147$.

[19] H. Dil, J. Lobo-Checa, R. Laskowski, P. Blaha, S. Berner, J. Osterwalder, T. Greber, Science 2008, 319, 1824. doi:10.1126/ SCIENCE. 1154179

[20] S. Berner, M. Corso, R. Widmer, O. Groening, R. Laskowski, P. Blaha, K. Schwarz, A. Goriachko, H. Over, S. Gsell, Angew. Chem., Int. Ed. 2007, 46, 5115. doi:10.1002/ANIE.200700234

[21] B. Wang, M.-L. J. Bocquet, Phys. Chem. Lett. 2011, 2, 2341. doi:10.1021/JZ201047C

[22] E. Bekyarova, S. Sarkar, F. Wang, M. F. Itkis, I. Kalinina, X. Tian, R. C. Haddon, Acc. Chem. Res. 2013, 46, 65. doi:10.1021/AR300177Q

[23] S. M. Avdoshenko, I. N. Ioffe, G. Cuniberti, L. Dunsch, A. A. Popov, ACS Nano 2011, 5, 9939. doi:10.1021/NN203719A

[24] M. Zhou, Y.-H. Lu, Y.-Q. Cai, C. Zhang, Y.-P. Feng, Nanotechnology 2011, 22, 385502. doi:10.1088/0957-4484/22/38/385502

[25] M. Zhou, A. Zhang, Z. Dai, C. Zhang, Y.-P. Feng, J. Chem. Phys. 2010, 132, 194704. doi:10.1063/1.3427246

[26] M. Zhou, A. Zhang, Z. Dai, Y.-P. Feng, C. Zhang, J. Phys. Chem. C 2010, 114, 16541. doi:10.1021/JP105368J

[27] X. Zhang, L. Yu, X. Wu, W. Hu, Adv. Sci. 2015, 2, 1500101. doi:10.1002/ADVS.201500101

[28] P. V. Avramov, A. A. Kuzubov, S. Sakai, M. Ohtomo, S. Entani, Y. Matsumoto, H. Naramoto, N. S. Eleseeva, J. Appl. Phys. 2012, 112, 114303. doi: $10.1063 / 1.4767134$

[29] A. L. Buchachenko, V. L. Berdinsky, Chem. Rev. 2002, 102, 603. doi:10.1021/CR010370L

[30] S. J. Chae, F. Günes, K. K. Kim, E. S. Kim, G. H. Han, S. M. Kim, H.-J. Shin, S.-M. Yoon, J.-Y. Choi, M. H. Park, C. W. Yang, D. Pribat, Y. H. Lee, Adv. Mater. 2009, 21, 2328. doi:10.1002/ADMA.200803016

[31] R. Rosei, M. Decrescenzi, F. Sette, C. Quaresima, A. Savoia, P. Perfetti, Phys. Rev. B 1983, 28, 1161. doi:10.1103/PHYSREVB. 28. 1161

[32] Y. Gamo, A. Nagashima, M. Wakabayashi, M. Terai, C. Oshima, Surf. Sci. 1997, 374, 61. doi:10.1016/S0039-6028(96)00785-6

[33] E. N. Voloshina, A. Generalov, M. Weser, S. Böttcher, K. Horn, Yu. S. Dedkov, New J. Phys. 2011, 13, 113028. doi:10.1088/1367-2630/13/ $11 / 113028$

[34] G. Bertoni, L. Calmels, A. Altibelli, V. Serin, Phys. Rev. B 2005, 71, 075402. doi:10.1103/PHYSREVB.71.075402

[35] G. Kalibaeva, R. Vuilleumier, S. Meloni, A. Alavi, G. Ciccotti, R. Rosei, J. Phys. Chem. B 2006, 110, 3638. doi:10.1021/JP055397D

[36] L. Adamska, Y. Lin, A. J. Ross, M. Batzill, I. I. Oleynik, Phys. Rev. B 2012, 85, 195443. doi:10.1103/PHYSREVB. 85.195443

[37] S. M. Kozlov, F. Viñes, A. Görling, J. Phys. Chem. C 2012, 116, 7360. doi:10.1021/JP210667F

[38] Yu. S. Dedkov, M. Fonin, New J. Phys. 2010, 12, 125004. doi:10.1088/ $1367-2630 / 12 / 12 / 125004$

[39] Yu. S. Dedkov, M. Fonin, New J. Phys. 2010, 12, 125004. doi:10.1088/ $1367-2630 / 12 / 12 / 125004$
[40] S. M. Kozlov, F. Viñes, A. Görling, J. Phys. Chem. C 2012, 116, 7360. doi:10.1021/JP210667F

[41] W. Zhao, S. M. Kozlov, O. Höfert, K. Gotterbarm, M. P. A. Lorenz, F. Viñes, C. Papp, A. Görling, H.-P. Steinrück, J. Phys. Chem. Lett. 2011, 2, 759. doi:10.1021/JZ200043P

[42] P. V. Avramov, S. Sakai, S. Entani, Y. Matsumoto, H. Naramoto, Chem. Phys. Lett. 2011, 508, 86. doi:10.1016/J.CPLETT.2011.04.016

[43] N. Ooi, A. Rairkar, J. B. Adams, Carbon 2006, 44, 231. doi:10.1016/ J.CARBON.2005.07.036

[44] N. Troullier, J. L. Martins, Phys. Rev. B 1992, 46, 1754. doi:10.1103/ PHYSREVB.46.1754

[45] J. H. Weaver, J. Luis Martins, T. Komeda, Y. Chen, T. R. Ohno, G. H. Kroll, N. Troullier, R. E. Haufler, R. E. Smalley, Phys. Rev. Lett. 1991, 66, 1741. doi:10.1103/PHYSREVLETT.66.1741

[46] M. B. Jost, N. Troullier, D. M. Poirier, J. L. Martins, J. H. Weaver, L. P. F. Chibante, R. E. Smalley, Phys. Rev. B 1991, 44, 1966. doi:10.1103/ PHYSREVB. 44.1966

[47] A. A. Kuzubov, E. A. Kovaleva, P. V. Avramov, A. V. Kuklin, N. S. Mikhaleva, F. N. Tomilin, S. Sakai, S. Entani, Y. Matsumoto, H. Naramoto, J. Appl. Phys. 2014, 116, 084309. doi:10.1063/1. 4894157

[48] P. V. Avramov, A. A. Kuzubov, S. Sakai, M. Ohtomo, S. Entani, Y. Matsumoto, H. Naramoto, N. S. Eleseeva, J. Appl. Phys. 2012, 112, 114303. doi:10.1063/1.4767134

[49] P. Hohenberg, W. Kohn, Phys. Rev. 1964, 136, B864. doi:10.1103/ PHYSREV.136.B864

[50] W. Kohn, L. J. Sham, Phys. Rev. 1965, 140, A1133. doi:10.1103/ PHYSREV.140.A1133

[51] J. P. Perdew, K. Burke, M. Ernzerhof, Phys. Rev. Lett. 1996, 77, 3865. doi:10.1103/PHYSREVLETT.77.3865

[52] G. Kresse, J. Hafner, Phys. Rev. B 1993, 47, 558. doi:10.1103/PHYS REVB. 47.558

[53] G. Kresse, J. Hafner, Phys. Rev. B 1994, 49, 14251. doi:10.1103/ PHYSREVB.49.14251

[54] G. Kresse, J. Furthmüller, Phys. Rev. B 1996, 54, 11169. doi:10.1103/ PHYSREVB.54.11169

[55] H. J. Monkhorst, J. D. Pack, Phys. Rev. B 1976, 13, 5188. doi:10.1103/ PHYSREVB.13.5188

[56] D. Hobbs, G. Kresse, J. Hafner, Phys. Rev. B 2000, 62, 11556. doi:10.1103/PHYSREVB.62.11556

[57] J. Lahiri, T. Miller, L. Adamska, I. I. Oleynik, M. Batzill, Nano Lett. 2011, 11, 518. doi:10.1021/NL103383B

[58] J. Lahiri, T. S. Miller, A. J. Ross, L. Adamska, I. I. Oleynik, M. Batzill, New J. Phys. 2011, 13, 025001. doi:10.1088/1367-2630/13/2/025001

[59] P. A. Khomyakov, G. Giovannetti, P. C. Rusu, G. Brocks, J. van den Brink, P. J. Kelly, Phys. Rev. B 2009, 79, 195425. doi:10.1103/ PHYSREVB.79.195425

[60] P. J. Feibelman, Phys. Rev. B 2008, 77, 165419. doi:10.1103/PHYS REVB.77.165419

[61] G. Bertoni, L. Calmels, A. Altibelli, V. Serin, Phys. Rev. B 2005, 71, 075402. doi:10.1103/PHYSREVB.71.075402

[62] J. P. Perdew, K. Burke, M. Ernzerhof, Phys. Rev. Lett. 1996, 77, 3865. doi:10.1103/PHYSREVLETT.77.3865

[63] A. Taylor, J. Inst. Met. 1950, 77, 585 .

[64] R. Rosei, M. Decrescenzi, F. Sette, C. Quaresima, A. Savoia, P. Perfetti, Phys. Rev. B 1983, 28, 1161. doi:10.1103/PHYSREVB. 28.1161

[65] Y. Gamo, A. Nagashima, M. Wakabayashi, M. Terai, C. Oshima, Surf. Sci. 1997, 374, 61. doi:10.1016/S0039-6028(96)00785-6

[66] G. Bertoni, L. Calmels, A. Altibelli, V. Serin, Phys. Rev. B 2005, 71, 075402. doi:10.1103/PHYSREVB.71.075402

[67] G. Kalibaeva, R. Vuilleumier, S. Meloni, A. Alavi, G. Ciccotti, R. Rosei, J. Phys. Chem. B 2006, 110, 3638. doi:10.1021/JP055397D

[68] L. Adamska, Y. Lin, A. J. Ross, M. Batzill, I. I. Oleynik, Phys. Rev. B 2012, 85, 195443. doi:10.1103/PHYSREVB.85.195443

[69] L. Yu. Antipina, P. V. Avramov, S. Sakai, H. Naramoto, M. Ohtomo, S. Entani, Y. Matsumoto, P. B. Sorokin, Phys. Rev. B 2012, 86, 085435. doi:10.1103/PHYSREVB. 86.085435 